\title{
Bruit de fond du tritium en milieu terrestre
}

\author{
S. Roussel-Debet ${ }^{\mathrm{a}}$ \\ IRSN, Pôle RadioProtection - Environnement, Service d'Étude et de Surveillance de la Radioactivité dans l'Environnement, Laboratoire \\ d'Étude Radioécologique en milieux Continental et Marin (PRP-ENV/SESURE/LERCM), Cadarache, 13115 St-Paul-lez-Durance, France.
}

Reçu le 19 juin 2013 - Accepté le 29 septembre 2013

\begin{abstract}
Résumé - Des résultats de mesures de tritium libre et organiquement lié ont été acquis de 2007 à 2012, en France métropolitaine, pour des échantillons de végétaux et de denrées prélevés en milieu terrestre, hors de l'influence potentielle d'un rejet. Le bruit de fond, déduit de ces données, n'évolue pas significativement; il est en moyenne de $1,4 \pm 1,2 \mathrm{~Bq} / \mathrm{L}$ pour le tritium libre et pour le tritium organiquement lié.
\end{abstract}

\begin{abstract}
Background levels of tritium in the terrestrial environment. Samples of tree leaves and terrestrial foodstuffs, obtained from French non-influenced zones were measured for tissue free water tritium (TFWT) and organically bound tritium (OBT) content, over the period 2007-2012. The background level determined from these data does not vary significantly over the 5 years of the survey and displays a mean value of $1.4 \pm 1.2 \mathrm{~Bq} / \mathrm{L}$ for both tritium forms.
\end{abstract}

Keywords: tritium / terrestrial environment / background levels

Une abondante littérature relative au comportement du tritium dans l'environnement et à sa modélisation a été publiée depuis plusieurs années, portant sur des recherches en laboratoire ou in situ, des méthodes de mesure, des synthèses de groupes nationaux ou internationaux (e.g., EU, 2008; ASN, $2010)$ et des articles plus opérationnels ( $c f$. notamment Belot et al., 1996; Boyer et al., 2009; Lebaron-Jacobs et al., 2009; Fournier et Jaunet, 2011; IAEA, 2012; Baglan et al., 2013; Guetat, 2013; Guetat et al., 2013). Cette note ne vise pas à reproduire la bibliographie existante, mais présente des résultats de mesure du tritium dans l'environnement non influencé par un rejet contemporain, afin de préciser le niveau du bruit de fond de ce radionucléide.

Hors de l'influence directe d'une installation rejetant du tritium, les concentrations du tritium, exprimées en $\mathrm{Bq} / \mathrm{L}$, sont globalement homogènes dans toutes les composantes du milieu terrestre (vapeur d'eau atmosphérique, eau de pluie, faune et flore), à l'exception des sols. Le bruit de fond cosmogénique dans l'hémisphère nord est de l'ordre de $0,6 \mathrm{~Bq} / \mathrm{L}$ dans l'eau de pluie, lié à une production naturelle évaluée à $72 \mathrm{PBq} / \mathrm{an}$ (UNSCEAR, 2000); la concentration de l'eau de pluie a atteint environ $470 \mathrm{~Bq} / \mathrm{L}$ en 1963 puis a considérablement diminué depuis la fin des essais militaires aériens jusqu'à un niveau de l'ordre de 5 à 10 Bq/L dans les années 1990 (IAEA/WMO, 2006; IRSN, 2009). Actuellement, ce bruit de fond fluctue aux alentours de 1 à $3 \mathrm{~Bq} / \mathrm{L}$. Cela correspond à une activité de l'air de l'ordre de $0,01 \mathrm{~Bq} / \mathrm{m}^{3}$ (tritium sous forme de vapeur d'eau) et à une activité massique des végétaux et animaux de

\footnotetext{
a sylvie.roussel-debet@irsn.fr
}

1 à $3 \mathrm{~Bq} / \mathrm{kg}$ frais. Le bruit de fond des eaux douces est de l'ordre de 1 à $3 \mathrm{~Bq} / \mathrm{L}$ et dix fois moindre, de 0,1 à $0,2 \mathrm{~Bq} / \mathrm{L}$, dans l'eau de mer.

Si l'on excepte les installations des sites CEA de Marcoule et Valduc (Tab. 1), aux alentours desquelles des niveaux de quelques dizaines à quelques centaines de $\mathrm{Bq} / \mathrm{L}$ sont régulièrement mesurés dans les échantillons biologiques, les eaux de surface et les eaux souterraines, l'influence locale, dans l'environnement terrestre, des rejets atmosphériques de tritium issus notamment des CNPE, est souvent imperceptible (Le Guen, 2008). Aussi, pour conforter l'expertise des mesures de tritium réalisées sur des échantillons prélevés au voisinage de ces installations, est-il utile de préciser la gamme actuelle du bruit de fond du tritium.

Des échantillons de végétaux prélevés à maturité (feuilles d'arbres, légumes), de vin et de produits animaux (viande, lait) ont été prélevés, de 2007 à 2012, sur des zones éloignées de toute installation nucléaire et non directement soumises à un rejet atmosphérique de tritium (Fig. 1). Au total, 32 échantillons de feuilles et 21 échantillons de denrées ont été prélevés. Le tritium organiquement lié (TOL) total, i.e., tritium de la matière sèche, échangeable et non échangeable, a été mesuré sur tous les échantillons, le tritium libre (HTO) sur 2/3 d'entre eux selon les méthodes schématisées figure 2 et brièvement décrites ci-après.

Pour chaque échantillon, environ un kilogramme frais est prélevé, placé dans un récipient hermétique et immédiatement mis en glacière. Dès l'arrivée au laboratoire, les échantillons sont lyophilisés. La matière sèche (une dizaine à une quarantaine de grammes) subit une combustion en flux d'oxygène et 
Tableau 1. Rejets de tritium en France en 2010 (ASN, 2013).

Tritium releases in France in 2010.

\begin{tabular}{cccc} 
& PBq & Contributeurs majeurs & $\begin{array}{c}\text { Ensemble des CNPE de EDF } \\
\text { (y compris Creys-Malville) }\end{array}$ \\
\hline Rejets gazeux & 0,75 & INBS de Marcoule et Valduc : & $4 \%$ \\
Rejets liquides & 11 & $\begin{array}{c}\text { Usine de retraitement de la Hague : } \\
89 \% \text { (rejet en Manche) }\end{array}$ & $9 \%$ \\
\hline
\end{tabular}

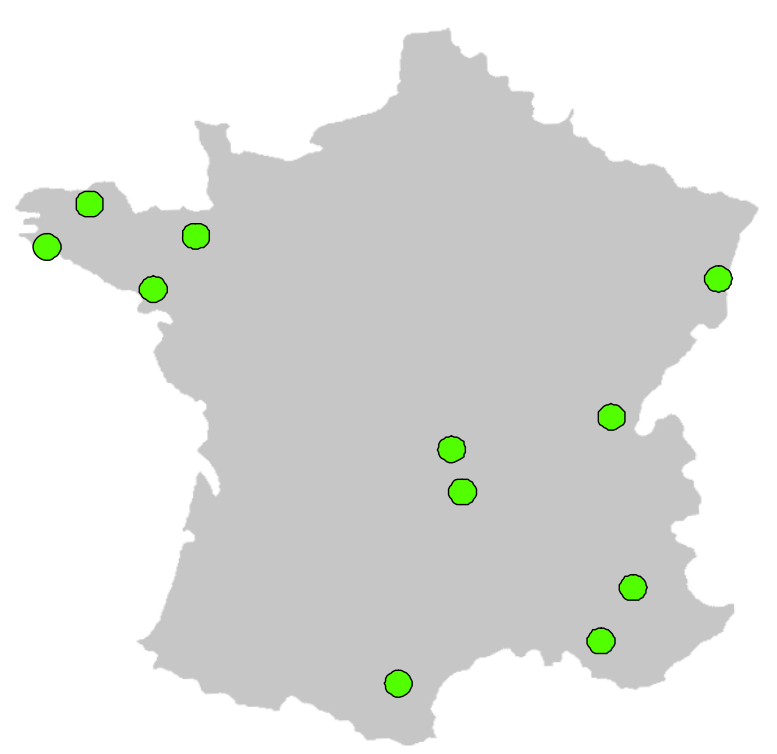

Fig. 1. Localisation des sites de prélèvement.

Localization of sampling sites.

d'argon. Le système de combustion (pyroxydizer) est constitué d'un tube de quartz glissé dans deux fours annulaires en série. Une pyrolyse est réalisée dans le premier four; les vapeurs sont envoyées sous un flux d'argon vers le second four où s'effectue une oxydation à $850^{\circ} \mathrm{C}$. Les vapeurs d'eau de combustion sont récupérées en sortie de four par un piège froid; l'eau de combustion recueillie est éventuellement neutralisée puis distillée à sec ; $10 \mathrm{~mL}$ sont mélangés à un scintillant pour une mesure en scintillation liquide sur un compteur à bas bruit de fond avec un temps de comptage de 1000 minutes. Pour quelques échantillons, le tritium organiquement lié a été déterminé par croissance de l'hélium $3: 25$ grammes environ de matière sèche lyophilisée est dégazée sous vide et conditionnée dans un conteneur, garantissant une étanchéité totale durant 3 à 6 mois, temps nécessaire à la formation d'une certaine quantité d'hélium 3, mesurée ensuite par spectrométrie de masse. L'eau de lyophilisation (environ $50 \mathrm{~mL}$ ) est par ailleurs distillée à sec puis $10 \mathrm{~mL}$ sont conditionnés pour une mesure en scintillation liquide. Les limites de détection sont similaires, de l'ordre de $1,3 \mathrm{~Bq} / \mathrm{L}$.

Les résultats sont reportés figure 3. Les activités étant faibles, un pourcentage non négligeable de résultats inférieurs aux limites de détection est observé : pour le tritium libre, respectivement $11 \%$ et $19 \%$ des échantillons de feuilles d'arbres

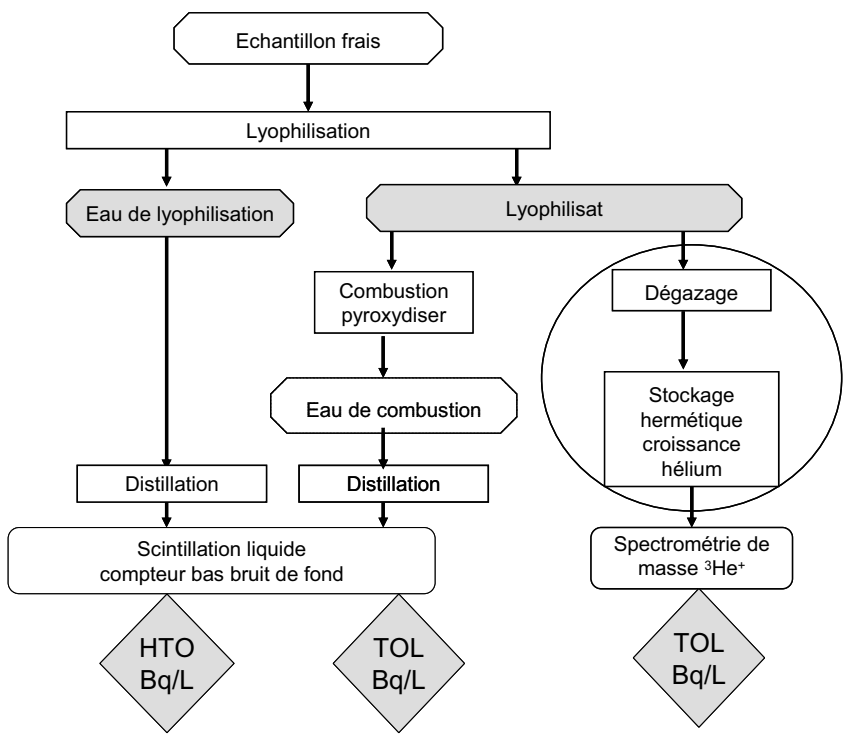

Fig. 2. Schéma des techniques de traitement et de mesure. L'échantillon frais est lyophilisé. L'eau de lyophilisation est distillée puis mesurée par scintillation liquide. Le lyophilisat est traité soit par combustion puis mesure par scintillation liquide (Cossonnet et al., 2009) soit dégazé et stocké pour permettre la croissance de l'hélium 3, ensuite mesuré par spectrométrie de masse (Jean-Baptiste et al., 2010). Les masses d'échantillons et les durées de comptage (ou de stockage pour la croissance de l'hélium 3) sont adaptées pour obtenir des limites de détection de l'ordre de 1,2 Bq/L.

Schematic diagram of the sample preparation and measurements. The fresh sample is freeze-dried. The water is distilled then measured by liquid scintillation counting. The dry residue is either burnt and then measured by scintillation (Cossonnet et al., 2009) or degassed then stored to allow for the accumulation of the helium-3, which is subsequently measured by mass spectrometry (Jean-Baptiste et al., 2010). The masses of samples and the time of counting (or storage for helium-3 ingrowth) were adapted to obtain limits of detection approximately equal to $1.2 \mathrm{~Bq} / \mathrm{L}$.

et de denrées sont inférieurs aux limites de détection et ces pourcentages sont plus élevés pour le tritium organiquement lié : $41 \%$ (feuilles) et $75 \%$ (denrées). Les valeurs obtenues ne montrent plus aucune diminution du tritium en fonction du temps (Tab. 2). Le rapport TOL/HTO des échantillons pour lesquels la mesure de HTO est supérieure à la limite de détection est variable, allant de 0,3 à 2,1. Cette variabilité du rapport, déjà observée, y compris pour les échantillons représentatifs du bruit de fond (e.g., Jean-Baptiste et al., 2011), peut avoir de multiples causes : fluctuations du métabolisme 
Tableau 2. Valeurs moyennes du tritium, dans des feuilles d'arbres et dans des denrées, hors de l'influence d'un rejet. $\mu$ : moyenne arithmétique ; $\sigma$ : écart-type arithmétique ; $n:$ nombre d'échantillons analysés (valeurs supérieures aux limites de détection).

Mean annual concentration of tritium in tree leaves and foodstuffs, in non-influenced zones. $\mu$ : arithmetic mean; $\sigma:$ standard deviation ; $n$ : number of measured samples (values superior to detection limits).

\begin{tabular}{|c|c|c|c|c|c|c|}
\hline \multirow[t]{2}{*}{ Année } & \multicolumn{3}{|c|}{$\begin{array}{c}\text { TOL } \\
\mathrm{Bq} / \mathrm{L} \text { d'eau de combustion } \\
\end{array}$} & \multicolumn{3}{|c|}{$\begin{array}{c}\text { HTO } \\
\mathrm{Bq} / \mathrm{L} \text { d'eau de cryodessiccation } \\
\end{array}$} \\
\hline & $\mu$ & $\sigma$ & $n$ & $\mu$ & $\sigma$ & $n$ \\
\hline 2007 & 1,1 & 0,2 & 4 & 1,4 & 0,4 & 4 \\
\hline 2008 & 1,5 & 0,6 & 33 & 1,4 & 0,6 & 10 \\
\hline 2009 & 1,0 & 0,5 & 6 & 1,8 & 0,9 & 4 \\
\hline 2011 & 1,4 & 0,7 & 14 & 1,4 & 0,7 & 14 \\
\hline 2012 & 1,6 & 0,4 & 4 & 1,0 & 0,3 & 4 \\
\hline 2007-2012 & 1,4 & 0,6 & 61 & 1,4 & 0,6 & 22 \\
\hline
\end{tabular}

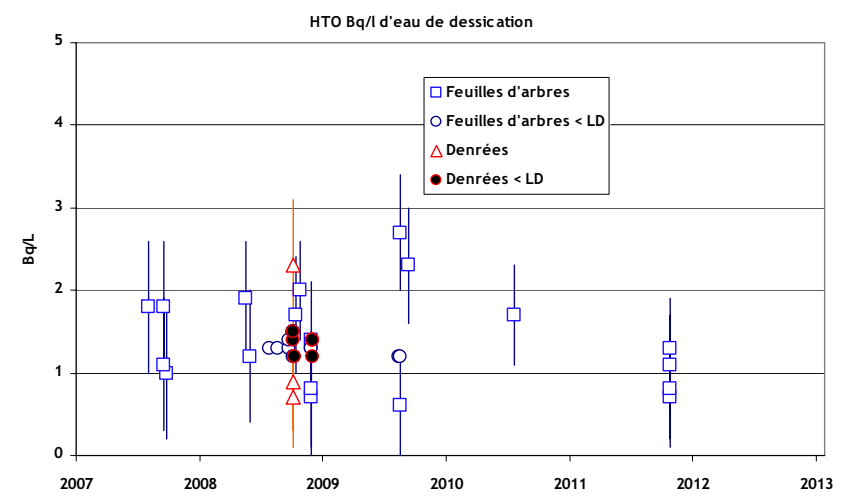

TOL Bq/l d'eau de combustion

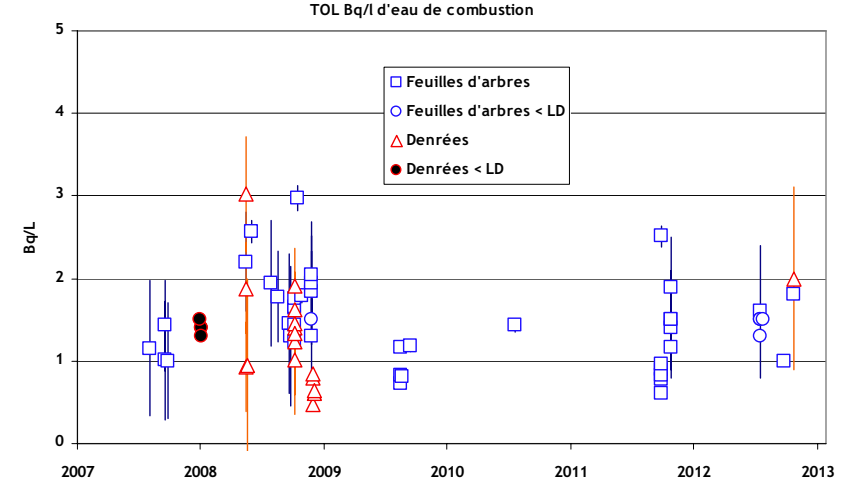

Fig. 3. Résultats de mesure du tritium dans les échantillons.

Tritium measurement results obtained in the samples.

végétal, des végétaux et des concentrations de la vapeur d'eau atmosphérique ou de la pluie, influence du sol... Cependant, on ne note pas de différence significative entre les concentrations moyennes en tritium libre et organiquement lié (test de Student, $\alpha=0,05)$.

La valeur du bruit de fond moyen, hors influence d'un rejet, déduit de la série complète d'échantillons 2007-2012 est de $1,4 \pm 1,2 \mathrm{~Bq} / \mathrm{L}$ pour le tritium libre et lié, soit une gamme de variation comprise entre la limite de détection et 2,6 Bq/L. Les résultats de mesures réalisées à proximité d'une installation ayant des rejets atmosphériques de tritium relativement faibles (moins d'une dizaine de $\mathrm{TBq} / \mathrm{an}$ ) induisant un surcroit de tritium minime peuvent ainsi être comparés à une gamme de référence hors influence. Cette comparaison doit tenir compte de la variabilité naturelle et des éventuels biais de traitement et de mesure des échantillons prélevés sur la zone potentiellement influencée que l'on étudie.

Remerciements. Les prélèvements et les prétraitements des échantillons ont été effectués par Gilles Salaun (IRSN/PRPENV/SESURE/LERCM). Le Laboratoire de Mesure de la Radioactivité dans l'Environnement (IRSN/PRP-ENV/STEME/LMRE) et le Laboratoire des Sciences du Climat et l'Environnement (LSCE, CEA-CNRS) ont réalisé les mesurages du tritium.

\section{Références}

ASN (2010) Livre blanc du tritium. Groupes de réflexion menés de mai 2008 à avril 2010 sous l'égide de l'ASN.

ASN (2013) Synthèse de l'inventaire des rejets de tritium, http:// livre-blanc-tritium.asn.fr

Baglan N., Kim S.B., Cossonnet C., Croudace I.W., Fournier M., Galeriu D., Warwick P.E., Momoshima N., Ansoborlo E. (2013) Organically bound tritium (OBT) behaviour and analysis: Outcomes of the seminar held in Balaruc-les-Bains in May 2012, Radioprotection 48 (1), 127-144.

Belot Y., Roy M., Métivier H. (1996) Le tritium de l'environnement à l'Homme. Les éditions de physique, Les Ulis.

Boyer C., Vichot L., Fromm M., Losset Y., Tatin-Froux F., Guetat Ph., Badot P.M. (2009) Tritium in plants: A review of current knowledge, Environ. Exp. Bot. 67 (1), 34-51.

Cossonnet C., Neiva-Marques A.M., Gurriaran R. (2009) Experience acquired on environmental sample combustion for organically bound tritium measurement, Appl. Radiat. Isotopes 67 (5), 809811.

EU (2008) European Commission, Emerging Issues on Tritium and Low Energy Beta Emitters. Dans : Proceedings of a scientific seminar, 13 November 2007, Luxembourg. Radiation. Protection No 152.

Fournier M., Jaunet P. (2011) Two years of a pluralistic work: ASN published a white paper on tritium, Radioprotection 46 (6), S627-S632.

Guetat Ph. (2013) Key mechanisms for tritium transfer in the terrestrial environment, Radioprotection 48 (2), 259-276.

Guetat Ph., Le Goff P., Boyer C., Duda J.M. (2013) Apports de la surveillance du centre CEA Valduc sur la connaissance des transferts de l'eau tritiée atmosphérique dans les différents compartiments de l'environnement, Radioprotection 48 (3), 367-389. 
IAEA (2012) Environmental Modelling for radiation Safety (EMRAS), A summary report of the results of the EMRAS Programme (2003-2007), Modelling the Environmental Transfer of tritium and Carbon-14 to biota and Man, IAEA-TECDOC1678.

IAEA/WMO (2006) Global Network of Isotopes in Precipitation. The GNIP Database. http://www.iaea.org/water.

IRSN (2009) Le tritium dans l'environnement. Rapport DEI 2009-05.

Jean-Baptiste P., Fourré E., Dapoigny A., Baumier D., Baglan N., Alanic G. (2010) ${ }^{3} \mathrm{He}$ mass spectrometry for very low-level measurement of organic tritium in environmental samples, J. Environ. Radioact. 101, 185-190.
Jean-Baptiste P., Fourré E., Baumier D., Dapoigny A. (2011) Environmental OBT/TFWT ratios revisited, Fusion Sci. Technol. 60, 1248-1251.

Lebaron-Jacobs L., Garnier-Laplace J., Lopez B., Adam-Guillermin C., Dublineau I., Roussel-Debet S., Antonelli C., Fievet B., Bailly du Bois P., Masson M. (2009) Tritium. Dans : Tritium, Toxicologie nucléaire environnementale et humaine. Chapitre 30 pp. 575-602. Éditions Tec\&Doc - Lavoisier, Paris.

Le Guen B. (2008) Impact du tritium autour des centrales nucléaires EDF, Radioprotection 43 (3), 467-467.

UNSCEAR (2000) Sources and Effects of Ionizing Radiation. Annex B: Exposures from natural radiation sources.

Cite this article as: S. Roussel-Debet. Bruit de fond du tritium en milieu terrestre. Radioprotection 49(2), 139-142 (2014). 